\title{
Citologia anal para RASTREAMENTO de LESÕES PRÉ-NEOPLÁsicas
}

\author{
Sidney Roberto Nadal*, Edenilson Eduardo Calore, luis Roberto Manzione Nadal, Sérgio Henrique Couto horta, Carmen \\ Ruth Manzione \\ Trabalho realizado pelas equipes técnicas de Proctologia e Patologia do Instituto de Infectologia Emílio Ribas, São Paulo, e Liga de Coloproctologia da \\ Faculdade de Ciências Médicas da Santa Casa de São Paulo.
}

* Correspondência:

Rua Dr. Virgílio de Carvalho

Pinto, 381, apto. 23

São Paulo/SP

Cep: 05415-030

Tel/fax: (11) 3337-4282

srnadal@terra.com.br

\section{RESUMO}

Oвjetıvo. Avaliar se a citologia anal com escova pode servir para rastreamento das lesões clínicas e subclínicas provocadas pelo HPV.

Métodos. Colhemos citologia anal, com escova, do canal anal de 102 doentes HIV-positivo com queixas proctológicas. Eram 86 homens e 16 mulheres com média etária de 37 anos. Destes, 33 negavam infecção pelo HPV, 14 haviam tratado verrugas, 28 tinham condilomas externos, sete apresentavam lesões internas e 20 os tinham em associação. 0 material foi enviado para exame de papanicolaou e coloração pela hematoxilina-eosina. Avaliamos as contagens de linfócitos T CD4+ para observar se o estado imunológico determinou as displasias mais avançadas.

Resultados. Somente um exame não pôde ser aproveitado. Os demais revelaram padrões celulares que variaram da normalidade até NIAa, incluindo a presença do HPV. Ocorreram 30 NIAs de baixo e 13 de alto grau em todos os grupos de doentes, com ou sem infecção pelo HPV. Em um dos doentes com NIAa e sem história prévia de infecção pelo HPV, e com úlcera no canal anal, a biópsia revelou carcinoma espinocelular invasivo. As médias de células T CD4+ nos portadores de NIA de baixo grau foi $281 / \mathrm{mm}^{3}$ e naqueles com NIAa foi 438/ $\mathrm{mm}^{3}$. A análise estatística mostrou diferença significante, revelando que, ao contrário do esperado, displasias menos acentuadas acometem doentes com contagens menores de linfócitos T CD4+. Esse fato demonstra que a imunidade sistêmica isolada parece não interferir na gênese dessas lesões, sugerindo que aspectos da imunidade local devam ser estudados. A avaliação estatística feita com a tabela $2 \times 2$ revelou sensibilidade de $74 \%$ e especificidade de $61 \%$.

Conclusão. Acreditamos que a citologia anal possa servir para esse rastreamento, selecionando os doentes para colposcopia anal e biópsias.

Unitermos: Infecções por Papilomavírus. Neoplasia intra-epitelial. Prevenção e controle. Canal anal. Citologia. Carcinoma de células escamosas. Sorodiagnóstico da Aids.

\section{INTRODUÇão}

Dentre os vários agentes etiológicos que provocam doenças na região perianal de indivíduos HIV-positivo, o Papilomavírus Humano (HPV) é o mais comum. A maioria das infecções pelo HPV não tem qualquer conseqüência clínica, mas cerca de $10 \%$ dos pacientes desenvolverão verrugas, papilomas ou displasias'. É também descrita a possibilidade de progressão dessas displasias, ou neoplasias intra-epiteliais anais (NIA), para carcinoma invasor ${ }^{2}$ e a maioria ocorreria na zona de transição do canal anal ${ }^{3}$.

O vírus, por si, não é suficiente para a carcinogênese, e a progressão tumoral, que ocorre em pequena porcentagem de indivíduos, pode ser estimulada por mutágenos químicos ou físi$\cos ^{\prime}$. Os fatores ligados ao desenvolvimento da NIA de alto (NIAa) e de baixo grau (NIAb), e à incidência elevada de recidivas dos condilomas, não estão completamente esclarecidos e a prevalência do HPV em lesões anogenitais de doentes HIV-positivo não é suficiente para explicar a grande proporção de NIAs neste grupo de enfermos ${ }^{4}$.
Vários fatores estão associados à maior probabilidade de desenvolvimento de displasia nos condilomas acuminados perianais: prática de sexo anal e soropositividade para HIV ${ }^{5-8}$, imunodepressão9, fases avançadas da infecção pelo $\mathrm{HIV}^{10}$, lesões verrucosas acima da linha pectínea ${ }^{7}$, contagens de linfócitos T CD4 inferiores a $500 / \mathrm{mm}^{3}$ 9,11-13, e tipo viral do HPV 1,14 . Entretanto, o tempo de aparecimento dos condilomas, tratamento tópico anterior e associação com outras doenças sexualmente transmissíveis não parecem representar fatores de risco ${ }^{7}$. Além disso, parece que o fator mais importante para o desenvolvimento do CEC anal é o tempo de infecção pelo HIVI5.

As NIAa são prováveis precursoras do tumor invasivo, com clara associação com os tipos de HPV de alto risco ${ }^{16}$. O risco de progressão pode estar ligado à severidade da $\mathrm{NIA}^{17}$ e, embora não haja comprovação, o tratamento dessas lesões preveniria a progressão para carcinoma ${ }^{16,18-20}$. NIA e câncer anal têm sido vistos com maior freqüência na junção escamocolunar, na linha pectínea ${ }^{21}$. Os fatores de risco entre os homens que praticam sexo com homens 
NADAL SR ET AL.

incluem 10 ou mais episódios de sexo anal receptivo durante a vida e a detecção de HPV anal. A prevalência de NIAa também é elevada entre doentes HIV-positivo usuários de drogas injetáveis, indicando que a infecção anal pelo HPV e as NIAs podem ser adquiridas mesmo pelos não praticantes do sexo anal receptivo ${ }^{22}$. Já as mulheres jovens adeptas dessa prática sexual e que têm passado de neoplasia intra-epitelial cervical (NIC) ou de infecção pelo HPV são consideradas as mais propensas ${ }^{23}$.

Devido ao risco do surgimento do tumor anal e a possibilidade da detecção das lesões precursoras, programas padronizados de rastreamento para a prevenção do câncer anal e protocolos de tratamento para NIA, em doentes infectados pelo HIV, deveriam ser instituídos ${ }^{16}$. $O$ uso dos esfregaços anais para citologia vem sendo realizado ${ }^{20,22,24-26} \mathrm{com}$ eficácia semelhante das coletas cervicais ${ }^{27}$, e com sensibilidade oscilando entre $42 \%$ e $98 \%$ e especificidade variando de $38 \%$ a $96 \%$, quando os resultados foram comparados com os da histologia, nos trabaIhos consultados $25,27,28$. A maioria dos autores indica a colposcopia anal para biópsias dirigidas quando a citologia mostra-se alterada ${ }^{20,26,29-31}$, e muitos outros recomendam-na como método de rastreamento para populações de alto risco para o carcinoma ana ${ }^{22,26,27,30}$.

A sensibilidade e a especificidade relatadas indicam que o método é aplicável para rastreamento de lesões subclínicas e com potencial oncogênico. Todavia, não encontramos na literatura consultada estudo que mostrasse a eficácia da citologia anal para detecção de lesões clínicas, uma vez que muitos profissionais não dispõem de anuscópio e que nem sempre o toque retal é suficiente para sugerir sua presença. Dessa forma, resolvemos colher material para citologia do canal anal, incluindo a linha pectínea, para avaliar se pode ser utilizado para rastreamento de lesões clínicas e subclínicas provocadas pelo HPV.

\section{Métodos}

O estudo é prospectivo, autorizado pelo Comitê de Ética em Pesquisa e pela Comissão Científica. Os procedimentos somente foram realizados após a assinatura do termo de consentimento livre e esclarecido.

Colhemos citologia anal, com escova, do canal anal de 102 doentes HIV-positivo com queixas proctológicas entre abril e junho de 2005. Eram 86 homens e 16 mulheres com média etária de 37 anos. Questionados quanto à presença de infecção anal pelo HPV, 33 negavam a doença, 14 referiram já terem erradicado as verrugas e 55 estavam em tratamento dos condilomas.

Colhemos material com escova que introduzimos no canal anal, até cerca de $4 \mathrm{~cm}$, tomando cuidado para não tocar nas verrugas da margem anal e evitar contaminação. Esfregamos em lâminas de vidro fazendo movimentos de rotação e em ziguezague para que toda a área fosse preenchida e todo o material pudesse entrar em contato com o vidro. Posteriormente, acomodamos as lâminas em recipiente plástico apropriado com álcool etílico a 70\% para fixação e enviamos para exame de papanicolaou e coloração pela hematoxilina-eosina e leitura.

Após essa coleta, submetemos os doentes a exame proctológico, incluindo anuscopia para avaliar a ocorrência de condilomas no canal anal. Não encontramos lesões clínicas nos 33 doentes que negavam sua presença e naqueles que referiram que a doença já havia sido tratada. Dentre os 55 que estavam em tratamento, 28 tinham condilomas externos, sete apresentavam lesões internas e 20 os tinham em associação. Avaliamos as contagens de linfócitos T CD4+ para observar se o estado imunológico determinou as displasias mais avançadas. Submetemos os resultados à análise estatística, com hipótese de nulidade de 5\% e a tabela $2 \times 2$ para avaliar a sensibilidade e a especificidade do método.

\section{Resultados}

A coleta dos exames transcorreu sem intercorrências. Os doentes negaram desconforto ou sangramento após o procedimento. A pouca celularidade encontrada impediu a leitura de lâmina de doente sem história de infecção pelo HPV. Os demais (99\%) revelaram padrões celulares que variaram da normalidade até NIAa, incluindo a presença do HPV. Na Tabela I, podemos observar $32 \mathrm{NIAb}, 14$ $\mathrm{NIAa}$ e três alterações nucleares inconclusivas (ANI) em todos os grupos, com ou sem infecção pelo HPV. Analisando cada tópico em separado, notamos que a citologia foi positiva em todos os casos com condilomas internos, porém em $70 \%$ dos doentes com lesões do canal e da margem anal. Dessa forma, a positividade para lesões clínicas do canal anal foi de 77,8\%. A citologia mostrou-se alterada em $38,7 \%$ dos doentes sem verrugas internas ou com lesões tratadas ou sem história de infecção pelo HPV, revelando mais NIAs naqueles com verrugas anteriormente tratadas (68,3\%).

Um dos doentes com NIAa, e sem história prévia de infecção pelo HPV, apresentava úlcera assintomática no canal anal cuja biópsia revelou carcinoma espinocelular invasivo.

A média dos linfócitos T CD4+ séricos nos portadores de $\mathrm{NIAb}$ foi $28 \mathrm{I} / \mathrm{mm}^{3}$ e naqueles com NIAa foi $438 / \mathrm{mm}^{3}$. A análise estatística mostrou diferença significante (t de Student, $p=0,038$ ).

A avaliação estatística feita com a tabela $2 \times 2$ revelou sensibilidade de $74 \%$ e especificidade de $61 \%$.

\begin{tabular}{lcccccc}
\hline \multicolumn{6}{c}{$\begin{array}{c}\text { Tabela I - Distribuição de I02 doentes submetidos à citologia anal, } \\
\text { conforme a presença e a localização da infecção pelo HPV e o } \\
\text { padrão de celularidade observado }\end{array}$} \\
\hline Condilomas & NIAb & NIAa & ANI & Metaplasia & Coilócitos & Total \\
\hline Internos & 05 & 02 & & & 05 & 07 \\
Int + ext & 07 & 05 & 02 & & 09 & 20 \\
Externos & 07 & 02 & & & 08 & 28 \\
Semhistória & 05 & 04 & 01 & 01 & 05 & 33 \\
Tratados & 08 & 01 & & & 03 & 14 \\
\hline Total & 32 & 14 & 03 & 01 & 30 & 102 \\
\hline
\end{tabular}

ANI: alterações nucleares inconclusivas; NIAa: neoplasia intra-epitelial anal de alto grau; NIAb: neoplasia intra-epitelial anal de baixo grau 


\section{Discussão}

Programas padronizados de rastreamento para a prevenção do câncer anal e protocolos de tratamento de NIA deveriam ser instituídos em doentes de risco ${ }^{16}$, e acreditamos que a citologia anal possa servir para esse fim, selecionando os pacientes para colposcopia anal e biópsias, evitando a evolução para o carcinoma anal. Para candidatar técnicas que previnam a progressão das lesões precursoras, a padronização e a melhora dos métodos são essenciais $^{19,24}$. Dentre as existentes, a citologia e a histologia têm sido as mais usadas.

Todavia, falhas de viés intra e interobservadores foram observadas na interpretação da $\mathrm{NIA}^{17}$, colocando restrições ao método e justificando os resultados variados da literatura ${ }^{17}$. Além disso, a concordância entre as interpretações da citologia e da biópsia ocorreu entre $32 \%$ e $50 \%$ dos casos ${ }^{17,24}$, índices insuficientes para justificar a aplicação do método. Entretanto, há estudos comparando os dois métodos que observaram sensibilidade de $95 \%$ e especificidade de 50\% para prevenir a severidade da lesão histológica ${ }^{25}$.

Os artigos da literatura têm avaliado a presença das lesões subclínicas. Nosso objetivo de avaliar se a citologia anal é efetiva na detecção das lesões clínicas provocadas pelo HPV tem a intenção de auxiliar profissionais de saúde que lidam com doentes portadores da infecção pelo HIV e de outras doenças sexualmente transmissíveis, e que não dispõem de aparelhagem para exame minucioso do canal anal, no diagnóstico das verrugas dessa região, nem sempre identificadas pelo simples toque digital. Todavia, sempre que possível, a retoscopia deve ser realizada, porque em nosso estudo a citologia não detectou os condilomas de 22,2\% dos doentes. Em nossa casuística, observamos alteração citológica em $77,8 \%$ dos doentes que apresentavam verrugas internas e em 38,7\% daqueles que não apresentavam lesão visível. Em um doente desse último grupo, e que apresentava NIAa, a histologia revelou carcinoma espinocelular invasivo, corroborando com aqueles que sugerem biópsias dirigidas quando houver alteração citológica ${ }^{25,31}$.

Em nossa casuística, a presença de lesões detectadas pela citologia teve $74 \%$ de sensibilidade e $61 \%$ de especificidade, dados semelhantes à literatura, que revelou índices de sensibilidade entre 69\% e 98\% e de especificidade entre $16,3 \%$ e 50\% 20,25,27,28,32,36. Preocupa-nos nossa incidência de 22,2\% de falsos-negativos. Podem ter ocorrido problemas na coleta, na aplicação na lâmina, no meio para transporte, nos métodos de coloração e na leitura. Entretanto, o material foi insuficiente para leitura em $1 \%$ das lâminas, similar à literatura que 0 situou entre $1 \%$ e $8,5 \% 19,26,32,33$. Suspeitamos que possa ter havido falha na coleta, quando a escova não alcançou as lesões, embora o método de colheita tenha sido padronizado, incluindo a distância que 0 instrumento foi introduzido no canal anal. Talvez a realização de duas coletas diminua o índice de falso-negativos. Não achamos estudos a esse respeito na literatura revista.

Essas anormalidades citológicas são mais incidentes em doentes HIV-positivo, indicando maior risco para NIA provavelmente devido à imunodepressão ${ }^{34}$. As médias dos linfócitos T CD4+ séricos nos portadores de NIAb foi $28 \mathrm{I} / \mathrm{mm}^{3}$ e naqueles com NIAa foi 438/ $\mathrm{mm}^{3}$. A análise estatística mostrou diferença significativa revelando que, ao contrário do esperado, displasias menos acentuadas acometem doentes com contagens menores de linfócitos T CD4+. Esse fato demonstra que a imunidade sistêmica isolada parece não interferir na gênese dessas lesões, sugerindo que aspectos da imunidade local devam ser estudados. Entretanto, há autores sugerindo a citologia anal como método de rastreamento em homens HIV-positivo com contagens séricas desses linfócitos inferiores a $500 / \mathrm{mm}^{3}$, independendo da preferência sexual, pelo risco aumentado de carcinoma anal e seus precursores 22.

\section{ConClusão}

Há quem diga que a citologia anal seja método mais aplicável que a colposcopia para diagnóstico e seguimento, devendo ser usada mais amplamente em doentes de risco ${ }^{35}$. Todavia, o aspecto citológico nem sempre corresponde ao achado da biópsia ${ }^{20,25,31}$. Qualquer anormalidade à citologia indica a possibilidade de lesões de alto grau (NIAa) na avaliação histológica3!'. Daí a importância da colposcopia anal para definir os locais onde as biópsias serão feitas, o que confirmará o grau da lesão $20,26,36$. Entretanto, estudos adicionais são necessários para determinar a eficácia desse procedimento para prevenção do tumor em questão ${ }^{22}$. De qualquer forma, baseados nos resultados obtidos nessa casuística, acreditamos que a citologia anal possa servir para esse rastreamento.

\section{Conflito de interesse: não há.}

\section{SUMMARY}

\section{ANAL CYTOLOGY FOR SCREENING OF PRE-NEOPLASIC LESIONS}

BACKGROUND. High grade intra-epithelial neoplasias (HAIN) are probable precursors of anal carcinoma, with association to high-risk types of Human Papillomavirus (HPV). This progression could be related to severity of the dysplasia and, albeit not yet confirmed, treatment of these lesions would prevent the evolution to cancer. Standardization and improvement of screening methods should therefore be essential to treat or prevent precursor lesions, mainly in patients at risk such as seropositives to Human Immunodeficiency Virus (HIV). The aim of this study was to evaluate if anal cytology, with a cytobrush, could be useful to screen clinic and pre-clinic lesions provoked by HPV.

METHODS. Brushes were used to obtain smears from the anal canal of 102 HIV-positive patients with proctologic complaints. There were 86 males and 16 females with a mean age of 37 years. HPV infection was denied by 33 patients, 14 had treated anal warts in the past, 28 had condylomas in the anal verge, seven had internal clinical lesions and 20 had both internal and external condylomas. The smears were submitted to Pappanicolaou and hematoxilineosin stains to identify cytological changes including HAIN. T $\mathrm{CD} 4+$ lymphocyte counts were also evaluated to check if the immunologic status caused more advanced dysplasia.

RESULTS. One smear only proved insufficient. All the others revealed cellular patterns varying from normality to HAIN. Low 
NADAL SR ET AL.

grade AIN (LAIN) occurred in 30 and HAIN in 13 patients. One patient with HAIN, without a history of HPV infection in the past, presented an anal canal ulcer which at biopsy was diagnosed as invasive squamous-cell carcinoma. T CD4+ cells averaged 281/ $\mathrm{mm}^{3}$ for LAIN patients and 438/ $\mathrm{mm}^{3}$ for HAIN patients. Analyses disclosed a statistical difference, showing that despite expectations, more advanced dysplasias occurred in patients with higher counts of T CD4+ cells. This fact demonstrated that isolated systemic immunity did not seem to interfere in the genesis of these lesions, suggesting that aspects of local immunity should be studied. Statistical analyses by a $2 \times 2$ table revealed sensibility of $74 \%$ and specificity of $61 \%$.

CONCLUSION. Results suggest that cytology could be used to diagnose anal cancer precursors. [Rev Assoc Med Bras 2007; 53(2): $|47-5|]$

KeY wORDS: Papillomavirus infections. Intra-epithelial neoplasia. Prevention and control. Anal canal. Cytology. Carcinoma squamous cell. Acquired Immunodeficiency Syndrome.

\section{REFERÊNCIAS}

I. Wieland $U$, Pfister $H$. Papilloma virus in human pathology. In: Gross GE, Barrasso R, Human papilloma vírus infection: a clinical atlas. Berlin/ Wiesbaden: Ullstein Mosby GmbH \& Co. KG; 1997. p.I-I8.

2. Modesto VL, Gottesman L. Sexually transmitted diseases and anal manifestations of AIDS. Surg Clin North Am 1994;74:|433-64.

3. Fenger C. Anal canal tumors and their precursors. In: Rosen PP, Fechner RE, editors. Pathology annual. 1988, part I. Norwalk: Apleton and Lange; 1988. p.45-66.

4. van Landuyt C, Mougin C, Drobacheff C, Bernard C, Merle C, Lab M, et al. Anogenital papillomavirus lesions in human with or without HIV infection. Comparison of colposcopic, histopathological and virological results. Ann Dermatolol Venereol 1993;120:281-6.

5. Palefsky JM, Holly EA, Gonzales J, Lamborn K, Hollander H. Natural history of anal cytologic abnormalities and papillomavirus infection among homosexual men with group IV HIV disease. J AIDS 1992; 5: | 258-65.

6. Carter PS, De Ruiter A, Whatrup C, Katzet DR, Ewings P, Mindel A, et al. Human immunodeficiency virus infection and genital warts as risk factors for anal intraepithelial neoplasia in homosexual men. Br J Surg 1995;82:473-4.

7. Metcalf AM, Dean T. Risk of dysplasia in anal condyloma. Surgery 1995; | | 8:724-6

8. Holly EA, WHittemore AS, Aston DA, Ahn DK, Nickoloff B], Kristiansen J. Anal cancer incidence: genital warts, anal fissure or fistula, hemorrhoids and smoking. J Natl Cancer Inst 1989;81:|726-31.

9. Palefsky JM, Holly EA, Gonzales J, Lamborn K, Hollander H. Detection of human papillomavirus DNA in anal intraepithelial neoplasia and anal cancer. Cancer Res 1991:51:1014-9.

I 0. Nadal SR, Manzione CR, Galvão VM, Salim VR, Speranzini MB. Perianal diseases in HIV-positive patients compared with a seronegative population. Dis Colon Rectum 1999:42:649-54.

I I. Critchlow CW, Surawicz CM, Holmes KK, Kuypers J, Daling JR, Hawes $\mathrm{SE}$, et al. Prospective study of high grade anal squamous intraepithelial neoplasia in a cohort of homosexual men: influence of HIV infection, immunosuppression and human papillomavirus infection. AIDS 1995; 9: | 255-62

12. Kiviat N, Rompalo A, Bowden R, Galloway D, Holmes KK, Corey L et al. Anal human papillomavirus infection among human immunodeficiency virus-seropositive and seronegative men. J Infect Dis $|990 ;| 62: 358-6 \mid$.
13. Levy AJ. Anal carcinoma. In: Levy AJ. HIV infection and development of cancer. Washington (DC): ASM Press; 1994.

I 4. Xi LF, Critchlow CW, Wheeler CM, Koutsky LA, Galloway DA, Kuypers $\mathrm{J}$, et al. Risk of anal carcinoma in situ in relation to human papillomavirus type 16 variants. Cancer Res 1998;58:3839-44.

15. Fagan SP, Bellows CF 3rd, Albo D, Rodriquez-Barradas M, Feanny M, Awad SS, et al. Length of human immunodeficiency virus disease and not immune status is a risk factor for development of anal carcinoma. Am J Surg 2005; 190:732-5.

16. Kreuter A, Brockmeyer NH, Hochdorfer B, Weissenborn SJ, Stucker M, Swoboda J, et al. Clinical spectrum and virologic characteristics of anal intraepithelial neoplasia in HIV infection. J Am Acad Dermatol 2005;52:603-8.

17. Colquhoun P, Nogueras JJ, Dipasquale B, Petras R, Wexner SD Woodhouse S. Interobserver and intraobserver bias exists in the interpretation of anal dysplasia. Dis Colon Rectum 2003;46: I332-6.

18. Manzione CR, Nadal SR, Calore EE. Postoperative follow-up of anal condylomata acuminata in HIV-positive patients. Dis Colon Rectum 2003; $46:$ : $358-65$

19. Mathews WC, Sitapati A, Caperna JC, Barber RE, Tugend A, Go U. Measurement characteristics of anal cytology, histopathology, and high-resolution anoscopic visual Impression in an Anal Dysplasia Screening Program. I Acquir Immune Defic Syndr 2004; 37:1610-5.

20. Palefsky JM, Holly EA, Hogeboom CJ, Berry JM, Jay N, Darragh TM. Anal cytology as a screening tool for anal squamous intraepithelial lesions. J Acquir Immune Defic Syndr Hum Retrovirol 1997; |4:4|5-22.

2 I. Goldstone SE, Winkler B, Ufford LJ, Alt E, Palefsky JM. High prevalence of anal squamous intraepithelial lesions and squamous-cell carcinoma in men who have sex with men as seen in a surgical practice. Dis Colon Rectum 2001;44:690-8.

22. Piketty C, Darragh TM, Da Costa M, Bruneval P. Heard I, Kazatchkine $M D$, et al. High prevalence of anal human papillomavirus infection and anal cancer precursors among HIV-infected persons in the absence of anal intercourse. Ann Intern Med 2003:I38:453-9.

23. Moscicki AB, Hills NK, Shiboski S, Darragh TM, Jay N, Powell K et al. Risk factors for abnormal anal cytology in young heterosexual women. Cancer Epidemiol Biomarkers Prev 1999;8:173-8.

24. Lytwyn A, Salit IE, Raboud J, Chapman W, Darragh T, Winkler B, et al. Interobserver agreement in the interpretation of anal intraepithelial neoplasia. Cancer 2005;103:1447-56.

25. Arain S, Walts AE, Thomas P, Bose S. The anal pap smear: cytomorphology of squamous intraepithelial lesions. Cytojournal 2005:2:4.

26. Friedlander MA, Stier E, Lin O. Anorectal cytology as a screening tool for anal squamous lesions: cytologic, anoscopic, and histologic correlation. Cancer 2004;102:19-26.

27. Fox PA, Seet JE, Stebbing J, Francis N, Barton SE, Strauss S, et al. The value of anal cytology and human papillomavirus typing in the detection of anal intraepithelial neoplasia: a review of cases from an anoscopy clinic. Sex Transm Infect 2005;81:142-6.

28. Papaconstantinou HT, Lee AJ, Simmang CL, Ashfaq R, Gokaslan ST, Sokol S, et al. Screening methods for high-grade dysplasia in patients with anal condyloma. J Surg Res 2005; 127:8-13.

29. Vajdic CM, Anderson JS, Hillman RJ, Medley G, Grulich AE. Blind sampling is superior to anoscope guided sampling for screening for anal intraepithelial neoplasia. Sex Transm Infect 2005;81:415-8.

30. Varnai AD, Bollmann M, Griefingholt H, Speich N, Schmitt C, Bollmann $R$ et al. HPV in anal squamous cell carcinoma and anal intraepithelial neoplasia (AIN) Impact of HPV analysis of anal lesions on diagnosis and prognosis. Int J Colorectal Dis 2006:21:135-42.

3 I. Panther LA, Wagner K, Proper J, Fugelso DK, Chatis PA, Weeden W, et al. High resolution anoscopy findings for men who have sex with men: inaccuracy of anal cytology as a predictor of histologic high-grade anal intraepithelial neoplasia and the impact of HIV serostatus. Clin Infect Dis 2004:38: | 490-2.

32. Cranston RD, Darragh TM, Holly EA, Jay N, Berry JM, Costa MD, et al. Self-collected versus clinician-collected anal cytology specimens to 
diagnose anal intraepithelial neoplasia in hiv-positive men. J Acquir Immune Defic Syndr 2004;36:9I5-920.

33. Bakotic WL, Willis D, Birdsong G, Tadros TS. Anal cytology in an HIVpositive population: a retrospective analysis. Acta Cytol 2005;49:163-8.

34. Durante AJ, Williams AB, Da Costa M, Darragh TM, Khoshnood K, Palefsky JM. Incidence of anal cytological abnormalities in a cohort of human immunodeficiency virus-infected women. Cancer Epidemiol Biomarkers Prev 2003; 12:638-42.

35. Scholefield JH, Johnson J, Hitchcock A, Kocjan G, Smith JH, Smith PA, et al. Guidelines for anal cytology to make cytological diagnosis and follow up much more reliable. Cytopathology 1998;9:। 5-22.

36. de Ruiter A, Carter P, Katz DR, Kocjan G, Whatrup C, Northover J, et al. A comparison between cytology and histology to detect anal intraepithelial neoplasia. Genitourin Med 1994;70:22-5.

37. Durante AJ, Williams AB, Da Costa M, Darragh TM, Khoshnood K, Palefsky JM. Incidence of anal cytological abnormalities in a cohort of human immunodeficiency virus-infected women. Cancer Epidemiol Biomarkers Prev 2003;12:638-42.

\footnotetext{
Artigo recebido: I / /05/06 Aceito para publicação: 27/10/06
} 